\title{
Heurísticas para Atribuição de Espectro em Redes Ópticas Elásticas Baseadas em Perda de Capacidade Sob Múltiplas Rotas
}

\author{
Matheus Lôbo dos Santos, Raul C. Almeida Jr. e Danilo R. B. Araújo
}

\begin{abstract}
Resumo-Esse artigo propõe duas heurísticas para atribuição de espectro em redes ópticas elásticas, considerando uma adaptação do algoritmo de atribuição de espectro denominado Min Slot Continuity Capacity Loss (MSCL). As propostas fazem uso de um conjunto de múltiplas rotas, ordenadas a partir de métricas como distância ou ocupação. As heurísticas são denominadas $M S C L$ Sequencial e MSCL Combinado, relacionando a forma como elas calculam a perda de capacidade nas rotas. $O$ desempenho foi comparado com a heurística amplamente utilizada chamada First-Fit. Os resultados obtidos mostram a capacidade das heurísticas em melhorar a probabilidade de bloqueio, demonstrando a eficácia de sua utilização.
\end{abstract}

Palavras-Chave-MSLC, Gerenciamento de recursos, EON, Múltiplas Rotas.

Abstract-This paper proposes two heuristics for spectrum allocation in elastic optical networks, considering an adaptation of the spectrum allocation algorithm called Min Slot-Continuity Capacity Loss (MSCL). The proposals make use of a set of multiple routes, ordered on the basis of metrics such as distance or occupancy. The name given to the heuristics was MSCL Sequencial and MSCL Combinado, relating the way they use the routes. The performance was compared with the widely used heuristic called First-Fit. The results obtained show that heuristics are able to improve the probability of blocking, demonstrating the effectiveness of its use.

Keywords-MSLC, Resource Management, EON, Multiple Routes.

\section{INTRODUÇÃO}

Devido à pandemia causada pelo novo Coronavírus, e a necessidade de se manter em isolamento social, a demanda por Internet no tráfego residencial aumentou para suprir trabalho remoto, entretenimento, educação e comércio, acarretando em mudanças no núcleo da Internet [1]. Com isso, pesquisas são necessárias para atingir as novas aplicações e tecnologias que exigem uma maior taxa de transmissão e confiabilidade. A utilização de redes ópticas visam suprir essas demandas de mercado, aplicando novas estratégias em busca de reduzir o custo financeiro da aplicação [2]. Nas últimas décadas, a tecnologia WDM (Wavelenght Division Multiplexing) foi o modelo de redes mais utilizado, fazendo uso de canais com faixa de espectro fixo para a transmissão dos dados [3].

Matheus Lôbo dos Santos, Departamento de Eletrônica e Sistemas (DES) UFPE, Maceió, e-mail: matheus.lobo@ufpe.br; Raul C. Almeida Jr, Departamento de Eletrônica e Sistemas (DES), UFPE, Recife, e-mail: ralmeida.ufpe@gmail.com.; Danilo R. B. Araújo, Departamento de Computação, UFRPE, Recife, e-mail: araujo.danilo.r.b@gmail.com. Este trabalho foi parcialmente financiado por $\mathrm{CNPq}$.
Em [4] foi proposta uma nova arquitetura de rede flexível, chamada de redes ópticas elásticas (EON, do inglês Elastic Optical Network). Essa rede surge como alternativa às redes WDM para atender de forma mais efetiva demandas heterogêneas. Em comparação ao WDM, as EONs utilizam a banda espectral de forma mais eficiente. Essa eficiência decorre da utilização de um ou mais slots de frequência consecutivos, minimizando os desperdícios na quantidade de banda necessária [5]. Nas redes EON, o problema de acomodação das demandas que chegam à rede é conhecido como roteamento e alocação de espectro (RSA, do inglês Routing and Spectrum Assignment). Com esse problema, surgem algumas restrições necessárias para o gerenciamento do espectro, são elas: continuidade e contiguidade. A continuidade implica a utilização dos mesmos slots de frequência em todos os enlaces que formam a rota candidata. Já o conceito de contiguidade impõe que os slots sejam usados sequencialmente no espetro (nos casos em que são necessários mais que um único slot)[6].

Algumas heurísticas foram adaptadas do problema de roteamento e alocação de comprimento de onda (RWA, do inglês routing and wavelength assignment) para o RSA. Exemplos de heurísticas adaptadas são o Random e o First-Fit. Na heurística Random, são escolhidos conjuntos de slots aleatórios que estejam disponíveis. Já no First-Fit, uma lista de alocação é gerada e os conjuntos de slots são escolhidos de acordo com a ordem da lista [7]. Em [8] foi apresentado o algoritmo de alocação de espectro denominado menor perda de capacidade para a continuidade de slot (MSCL, do inglês Min Slot-Continuity Capacity Loss), cujo objetivo é analisar, em cada requisição, o estado dos caminhos ópticos na rede a fim de obter o melhor conjunto de slots para alocar a requisição, de modo que a perda de capacidade seja a menor possível, reduzindo dessa forma a probabilidade de bloqueio das requisições subsequentes.

Diversos trabalhos aplicaram essa heurística adaptada a novos problemas, como: aplicação em Fibras Multi-Núcleo [9]; combinado com a técnicas de Split Spectrum [10] e avaliando a fragmentação do espectro [11]. A fragmentação espectral [12] é um problema proveniente de requisições com diferentes tamanhos de demanda, sendo responsável por degradar o desempenho da rede. Dessa forma, boas heurísticas em EON devem buscar a compactação do uso de espectro, a fim de reduzir a fragmentação.

Este trabalho está organizando da seguinte forma: na Seção II, é realizada uma fundamentação do algoritmo MSCL. Na Seção III, é explicada como foi realizada a adaptação da heurística MSCL para o cenário de múltiplas rotas. Na Seção 
IV, são apresentadas as configurações utilizadas no processo de simulação. $\mathrm{Na}$ Seção $\mathrm{V}$, estão as simulações e as discussões dos resultados obtidos. Por fim, na Seção V, são apresentadas as considerações finais.

\section{Fundamentação TeÓricA - Algoritmo MSCL}

O MSCL é um algoritmo de alocação de espectro usado em EONs. Conforme detalhado em [9] e [10], sua premissa se baseia na escolha do melhor conjunto de slots que, ao serem alocados na rede, irão causar o menor impacto em termos de perda de capacidade de alocação. Para cada solicitação de requisição, é feito o cálculo da perda de capacidade considerando a rota principal da requisição a ser alocada, juntamente com todas as rotas que compartilham ao menos um enlace com ela. $\mathrm{O}$ valor da perda de capacidade para uma demanda de $n$ slots em uma rota $r$ é calculado por:

$$
C^{<r>}(n)=\sum_{p \in I_{r}} S^{<p>}(\psi, n)-S^{<p>}\left(\psi^{\prime}, n\right),
$$

em que: $C^{<r>}(n)$ é a perda de capacidade para requisição com $n$ slots após seu estabelecimento na rota $r ; I_{r}$ é o conjunto de rotas que interferem com a rota $r ; p$ é a rota interferente atual; $\psi$ representa o estado atual da rede (ou seja, o conjunto de todos os caminhos e slots alocados); $\psi^{\prime}$ representa o estado da rede após o estabelecimento da requisição; $S^{<p>}(\psi, n)$ é o número total de possibilidades de alocação que uma requisição por $n$ slots tem para alocar no caminho $p$ antes do estabelecimento de $r ; S^{<p>}\left(\psi^{\prime}, n\right)$ é o número total de possibilidades de alocação que uma requisição por $n$ slots tem para alocar no caminho $p$ após estabelecimento de $r$.

A Equação 1 calcula o impacto que uma requisição de tamanho $n$ tem sobre a rota $r$ e todas as que interferem com ela. Para obter a perda de capacidade total na rede é necessário levar em consideração todas as demandas possíveis para a rota $r$. Assim, o cálculo é dado por:

$$
C^{<r>}=\sum_{n \in N} C^{<r>}(n),
$$

em que $N$ é o conjunto de todas as possíveis classes de demandas que podem ser alocadas em $r$ e $C^{<r>}$ representa a perda de capacidade total para todo o conjunto $N$. A posição espectral que obtiver o menor $C^{<r>}$ obtido pela Equação 2 será a posição de alocação da requisição.

\section{Heurísticas Propostas}

As heurísticas propostas neste trabalho são uma adaptação do algoritmo MSCL para redes apresentadas em [9] e [10]. A principal diferença está na forma de escolher entre diversas perdas de capacidade em múltiplas rotas. A partir desse ponto, duas propostas são apresentadas. Em ambas, inicialmente são obtidas $K$ rotas de forma offline. A primeira proposta, nomeada de $M S C L$ Combinado, utiliza a Equação 2 para calcular a perda de capacidade em cada uma das $K$ rotas, fazendo uso daquela que apresenta a menor perda de capacidade do conjunto. Essa rota juntamente com o conjunto de slots que apresentarem a menor perda de capacidade serão usados para alocar a requisição. A segunda proposta, intitulada MSCL Sequencial, calcula a perda de capacidade para cada alocação das rotas em sequência. O MSCL Sequencial começa por ordenar as rotas por algum critério. Após isso, a perda de capacidade na primeira rota $(k=1)$ é calculada. Caso essa rota apresente recursos suficientes para a alocação, isto é, um conjunto de slots disponíveis para alocar a requisição, o conjunto de slots que provê a menor perda de capacidade nesta rota são usados. Caso não seja possível alocar em $k=1$, a tentativa é feita nas rotas com valores de $k$ subsequentes.

A Figura 1 apresenta a política de escolha de cada heurística abordada neste artigo para um certo instante da rede. Nesses cenários com $k=3$, foi exemplificada a perda de capacidade de 4 conjuntos de slots. O First-Fit, mostrado em azul, escolhe o primeiro conjunto disponível para a rota com $k=1$. O MSCL Sequencial, representado na cor amarela, escolhe o conjunto com a menor perda de capacidade para a rota $k=1$. O MSCL Combinado, por sua vez, é capaz de analisar todos os conjuntos de slots em cada uma das rotas, escolhendo a combinação que apresentar o menor impacto na rede.

\begin{tabular}{|l|c|c|c|c|}
\cline { 2 - 5 } \multicolumn{1}{c|}{} & \multicolumn{4}{c|}{ Conjunto de slots } \\
\cline { 2 - 5 } \multicolumn{1}{c|}{} & $\mathrm{A}$ & $\mathrm{B}$ & $\mathrm{C}$ & $\mathrm{D}$ \\
\hline Rota $\mathrm{k}=1$ & 58 & 64 & 54 & 56 \\
\hline Rota $\mathrm{k}=2$ & 65 & 50 & 60 & 57 \\
\hline Rota $\mathrm{k}=3$ & 72 & 74 & 85 & 78 \\
\hline First-Fit & \multicolumn{3}{|c|}{ MSCL S } & \multicolumn{3}{|c}{ MSCL C } \\
\hline
\end{tabular}

Fig. 1. Exemplo de alocação das heurísticas abordadas nesse artigo.

A maneira pela qual este trabalho calcula a perda de capacidade é baseada no número de formas em uma lacuna. O conceito de lacuna indica a existência de slots contíguos disponíveis em determinada rota, cujo tamanho é definido pela quantidade de slots que ele possui. Caso uma lacuna apresente tamanho superior à quantidade de slots necessários para acomodar a requisição, é possível encontrar o número de formas como um conjunto de $n$ slots pode ser alocado na lacuna. A Figura 2 demonstra um exemplo para uma rede com 10 slots e uma requisição por $n=2$ slots.

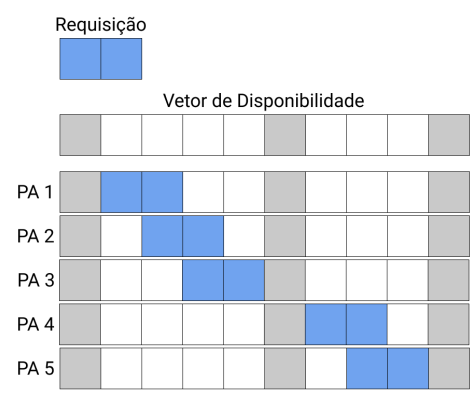

Fig. 2. Representação das possibilidades de alocação para uma requisição de 2 slots no vetor de disponibilidade.

Na Figura 2, os slots já alocados estão representados pela cor cinza. $\mathrm{Na}$ cor azul, são representados os slots para a requisição a ser alocada. Existem 5 possibilidades de alocação (PA 1 ao PA 5) para esse exemplo. Esse valor pode ser obtido 
conhecendo os tamanhos e as quantidades das lacunas. Nesse exemplo, há uma lacuna de tamanho 4 e outra de tamanho 3 . Assim, o número de formas é definido como:

$$
s(n)=\sum_{l \in L} s\left(h_{l}, n\right)=\sum_{l \in L}\left(h_{l}-n+1\right),
$$

em que: $s\left(h_{l}, n\right)$ é o número de formas como uma requisição de tamanho $n$ pode ser alocada em uma lacuna de tamanho $h_{l} ; L$ é o conjunto de todas as lacunas presentes no vetor de disponibilidade. A perda de capacidade é obtida por meio da comparação deste valor antes e depois de uma possível alocação, conforme mostrado na Equação 1. Por ser um problema $N P$-Hard não exite uma solução ótima, sendo possível obter uma sub-ótima por meio de heurísticas.

\section{PROCEDIMENTO EXPERIMENTAL}

Para avaliação das propostas deste trabalho, foram realizados experimentos computacionais auxiliados por um simulador de redes ópticas elásticas. O simulador utilizado é uma adaptação para EONs do simulador SIMTON [13]. Para permitir uma avaliação mais abrangente dos benefícios de cada algoritmo, foram consideradas duas topologias diferentes com links bidirecionais, conforme ilustram as Figuras 3 e 4 . Para cada simulação foram utilizadas $10^{5}$ requisições, com o tráfego de chegada assumindo um modelo Poissoniano e com duração das requisições distribuídas exponencialmente. Os impactos de camada física considerados nessa pesquisa foram o ruído ASE (do inglês, Amplified Spontaneous Emission) e ganho no amplificador óptico. Foram considerados os seguintes formatos de modulação: 4,8,16,32 e 64-QAM, com SNR de $6.8,8.6,10.5,12.6$ e 14.8 , respectivamente.

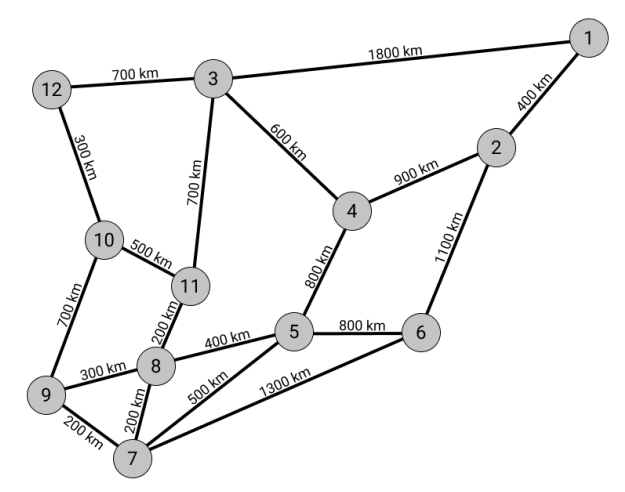

Fig. 3. Representação da topologia Finlândia com 12 nós e 38 links.

Os nós de origem e destino são escolhidos a partir de uma função de densidade de probabilidade Uniforme, assim como a taxa de bits sorteada por requisição. Essa taxa assume os seguintes valores: 50, 75, 100, 200 e 300 Gbits/s. Foram adotados 64 slots em cada enlace da topologia. Para o roteamento, foi escolhido o algoritmo Yen[14] para encontrar as $K$ menores rotas com base na métrica de distância. O desempenho dos algoritmos foi medido a partir da probabilidade de bloqueio (relação entre o número de requisições bloqueadas e o total de requisições enviadas) e do tempo de simulação. Os bloqueios, por sua vez, ocorrem quando não há um conjunto de slots

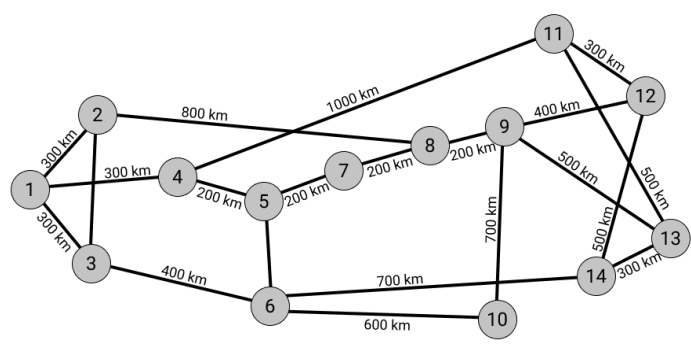

Fig. 4. Representação da topologia NSFNet com 14 nós e 42 links.

disponíveis para alocar a requisição com o formato de modulação de maior eficiência espectral que atenda os requisitos da camada física. O computador usado para as simulações é um i5-8265U de oitava geração com frequência de $1.6 \mathrm{GHz}$ e 8 núcleos, $8 \mathrm{~Gb}$ de mémoria RAM DDR4 de $2666 \mathrm{MHz}$, armazenamento com SSD padrão M2 e placa gráfica nvidia geforce $230 \mathrm{mx}$ de $1 \mathrm{~Gb}$.

O algoritmo escolhido para critério de comparação foi o First-Fit, por ser uma heurística bastante explorada e validada em diversas pesquisas com aplicações em redes WDM e EON. O First-Fit é um algoritmo simples e com baixo esforço computacional. Apresenta uma característica importante para redes EON no que diz respeito à compactação do espectro. Com isso, é capaz de fornecer bons resultados na busca por recursos espectrais em um baixo tempo de execução. Visando reduzir o tempo de simulação, o cálculo da probabilidade de bloqueio é finalizado antes de $10^{5}$ requisições, caso uma sequência de 1000 bloqueios ocorra. Esse valor é o suficiente para trazer confiabilidade à simulação.

\section{Resultados E Discuss Ão}

A Figura 5 apresenta os resultados obtidos em termos de probabilidade de bloqueio versus carga da rede para todos os algoritmos considerados no estudo. A Figura 5 (a) utiliza o roteamento fixo alternativo de caminho mais curto pela distância. Fazendo a comparação dos algoritmos para uma única rota $(k=1)$, os algoritmos MSCL Sequencial e Combinado apresentam o mesmo desempenho, conforme esperado pela natureza das suas heurísticas. Em todas as cargas simuladas, o desempenho dos algoritmos propostos apresentaram melhoria ao First-Fit. Observando as heurísticas em $k=2$, o First-Fit melhora o desempenho, superando até mesmo os algoritmos $M S C L$ com $k=1$. A melhora mais significativa é encontrada utilizando o MSCL Combinado, que na carga de 75 Erlangs, apresentou uma melhoria de $61.58 \%$ quando comparado ao First-Fit. Ao comparar o MSCL Sequencial com o First-Fit, a melhoria foi de $14.28 \%$. Para $k=3$ essa relação foi mantida, na proporção de $71.05 \%$ e $19.44 \%$, respectivamente.

Para evitar a suspeita de que a melhoria expressiva do $M S C L$ Combinado advém da distribuição de carga entre as rotas e não propriamente da alocação combinada rota e lacuna com menor perda de capacidade, foi feita uma análise que ordena as $K$ rotas antes da aplicação do First-Fit ou do MSCL Sequencial (o desempenho do MSCL Combinado é indiferente à ordenação ou não das rotas), dando-se prioridade à rota menos congestionada, conforme apresentado na Figura 5 (b). Para $k=1$, 


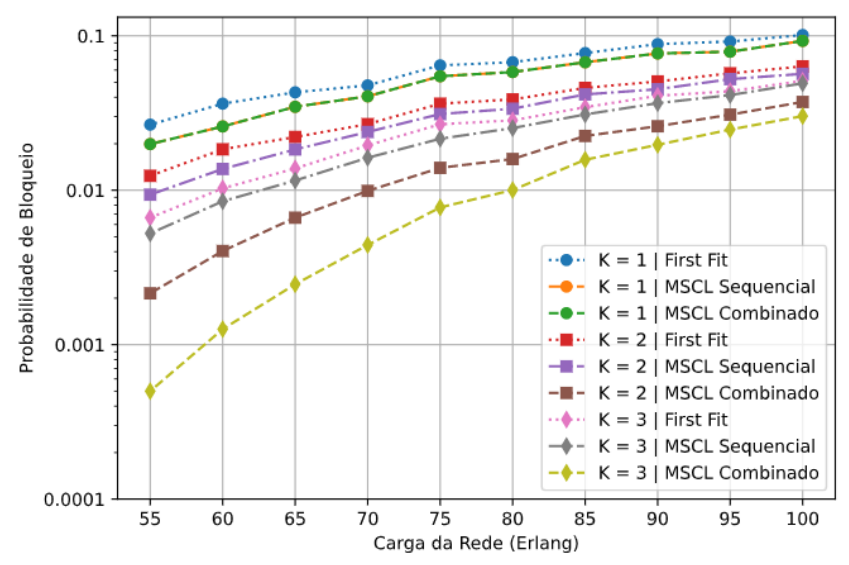

(a)

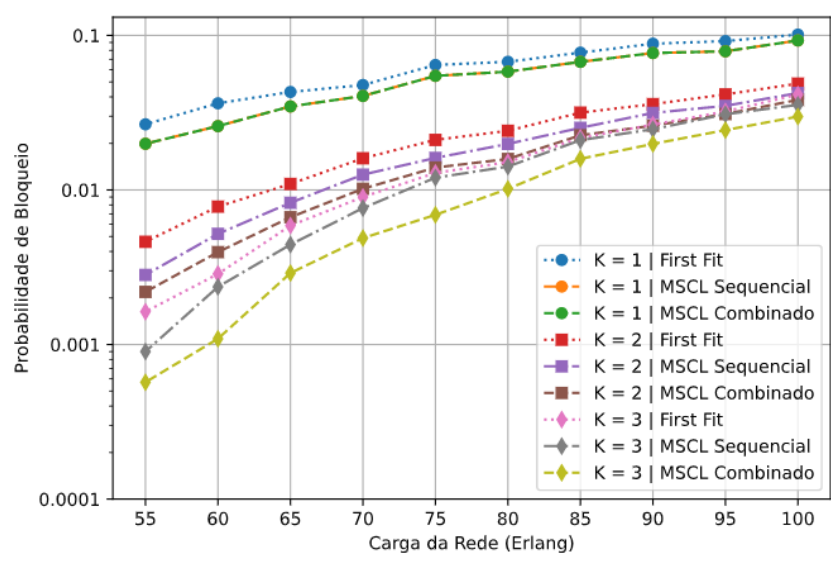

(b)

Fig. 5. Topologia Finlândia: (a) Comparativo entre a probabilidade de bloqueio vs carga da rede para $K$ rotas ordenadas a partir da menor distância; (b) Comparativo entre a probabilidade de bloqueio vs carga da rede para $K$ rotas ordenadas a partir da menor ocupação WDM;

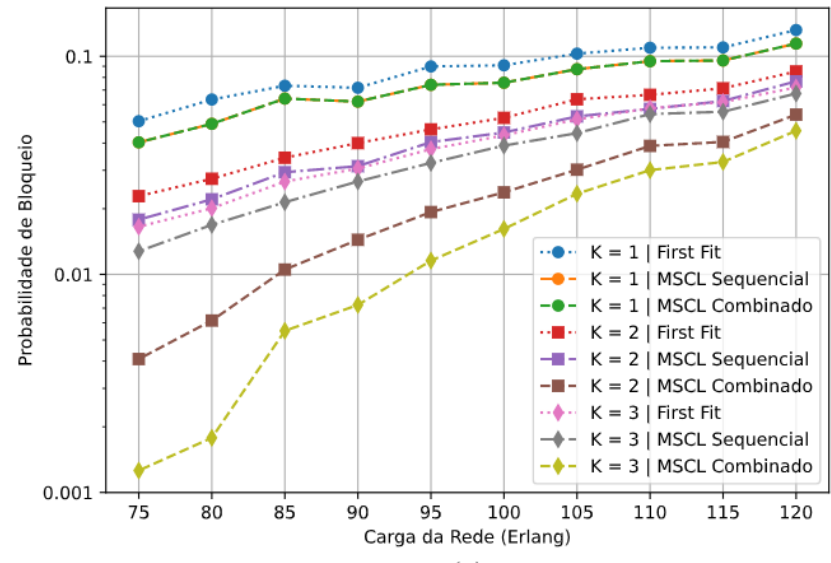

(a)

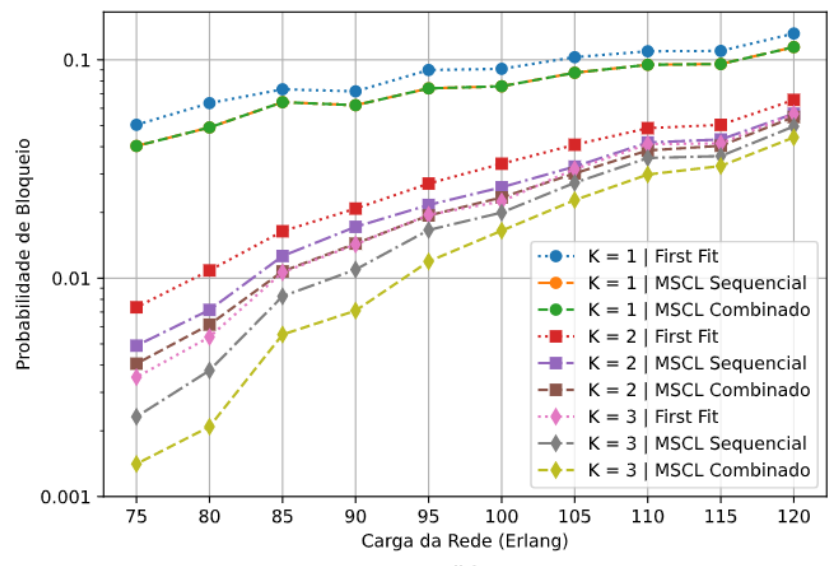

(b)

Fig. 6. Topologia NSFNet: (a) Comparativo entre a probabilidade de bloqueio vs carga da rede para $K$ rotas ordenadas a partir da menor distância; (b) Comparativo entre a probabilidade de bloqueio vs carga da rede para $K$ rotas ordenadas a partir da menor ocupação WDM;

o desempenho foi mantido, conforme esperado. Para $k=2$, as heurísticas propostas continuaram a apresentar melhorias em comparação ao First-Fit em todos os pontos simulados. $\mathrm{Na}$ carga de 75 Erlangs a melhoria em realação ao First-Fit foi de $23.53 \%$ e de $33.74 \%$ para o MSCL Sequencial e o Combinado, respectivamente. Para $k=3$, a consistência entre os algoritmos foi mantida, apresentando melhoria de $6.92 \%$ e $46.48 \%$, respectivamente. Empates ocorrido na menor perda de capacidade em diferentes rotas, são desempatados pelas rotas com a menor ocupação. Sendo assim, mesmo após aplicar um algoritmo que distribua a carga, as heurísticas propostas ainda se mantêm melhores que o First-Fit, para reduzir a probabilidade de bloqueio. Nesse caso, ambos os algoritmos propostos apresentam melhorias significativas comparados ao First-Fit. Ao dar preferência a uma rota menos congestionada, o MSCL Sequencial terá mais opções para fazer a escolha espectral.

Essas análises também foram realizadas para a topologia NSFNet, conforme mostrado na Figura 6 (a) para rotas ordenadas a partir da menor distância e na Figura 6 (b) para ordenamento de acordo com a menor ocupação. Nessa topologia, as diferenças entre os algoritmos propostos e o
First-Fit também mostraram-se consistentes, pois em nenhum ponto de carga o resultado é inferior ao desempenho do First-Fit. Na Figura 6 (b), para uma carga de 90 Erlangs e $k=2$ o ganho em relação ao First-Fit foi de $17.50 \%$ e $30.9 \%$ para os algoritmos MSCL Sequencial e Combinado, respectivamente. Analisando $k=3$, o Sequencial apresentou $23.29 \%$ de ganho, enquanto o Combinado $50.28 \%$. As ordens dos desempenhos dos algoritmos entre as topologias Finlândia e NSFNet mantiveram-se a mesma, concluindo-se que esses resultados devem ser mantidos para diferentes topologias.

A Figura 7 mostra o tempo de execução para simular as curvas mostrada na Figura 5 (b). Conforme o valor de $K$ aumenta, há um aumento da complexidade computacional. Um $K$ elevado implica em mais rotas a serem analisadas, como também pelo maior conjunto de rotas interferentes a cada rota analisada. O somatório na Equação 1 percorre todo o conjunto, a fim de obter o valor da perda de capacidade na rota principal e suas interferentes. Sendo assim, é possível notar o tempo de execução aumentando em ordens de grandeza para os algoritmos MSCL. Em contraponto, um valor de $K$ elevado permite que o algoritmo MSCL Combinado analise uma maior diversidade de rotas, podendo tomar a decisão de alocar em 


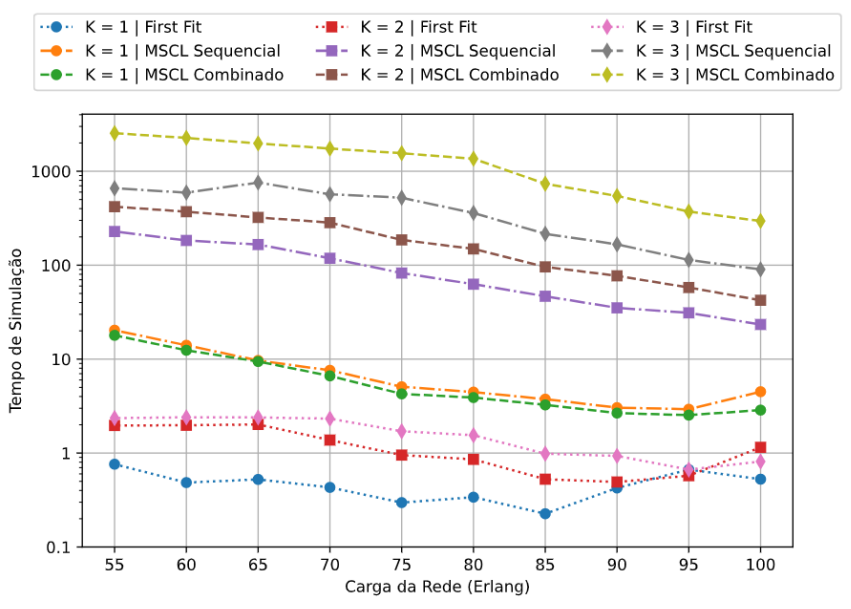

Fig. 7. Tempo de simulação (segundos) versus carga da rede (Erlangs) para a topologia Finlândia.

uma rota mais longa, porém com menor perda de capacidade, caso ela apresente uma menor perda de capacidade.

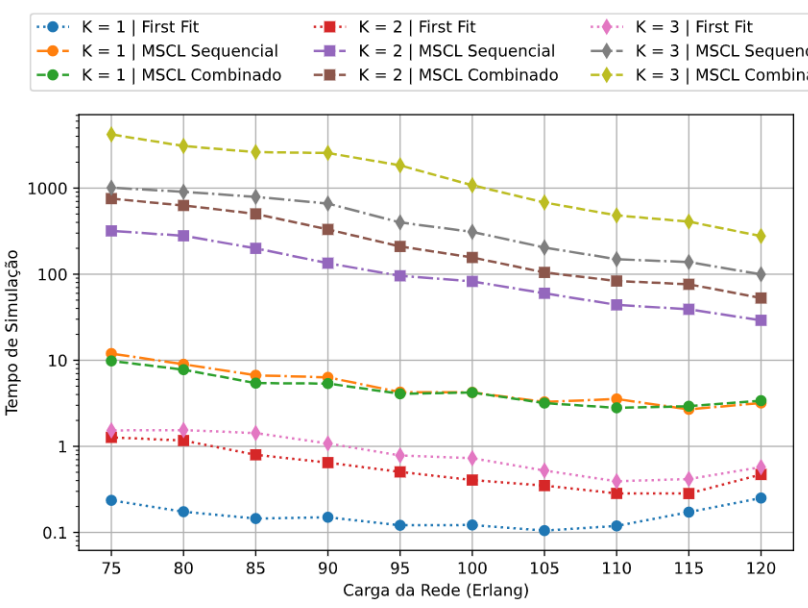

Fig. 8. Tempo de simulação (segundos) versus carga da rede (Erlangs) para a topologia NSFNet.

A Figura 8 mostra o tempo de execução para simular as curvas mostradas na Figura 6 (b). De forma semelhante ao ocorrido com as probabilidades de bloqueio, o tempo de simulação permaneceu na mesma ordem em ambas as topologias. Foi observado também durante a pesquisa que rotas menores em número de enlaces apresentam um menor número de rotas interferentes. A Equação 1 define um somatório em termos do conjunto interferente, ou seja, comparar cálculos de perda de capacidade em conjuntos interferentes com diferentes número de rotas acaba por não ser totalmente justo. Contudo, as menores tendem a ser mais escolhidas de forma que seu papel é cumprido. Esse critério de comparação merece ser estudo em trabalhos futuro. Embora a NSFNet possua mais nós e mais enlaces quando comparada à Finlândia, o tempo de simulação foi muito próximo analisando os mesmos algoritmos.

\section{CONCLUSÕES}

Nesse artigo, foram introduzidas duas heurísticas para atribuição do espectro em redes ópticas. As heurísticas foram adaptadas do conceito de MSCL desenvolvido para redes EON e moldado para utilização em múltiplas rotas. Ambos os algoritmos têm como objetivo a redução do impacto causado pela perda de capacidade após a alocação do espectro, tanto na rota escolhida para a requisição quanto em todas suas rotas interferentes. A proposta foi comparada com a heurística FirstFit considerando a probabilidade de bloqueio em conjuntos com uma, duas e três rotas. Ao término das simulações, ficou comprovada a redução na probabilidade de bloqueio da heurística MSCL Sequencial em relação ao First-Fit e $M S C L$ Combinado em relação ao First-Fit. Para a topologia Finlândia, essa última relação apresentou melhoria de $71.05 \%$ no roteamento realizado com a distância. Considerado o roteamento por ocupação, essa melhoria foi de $46.48 \%$ e $50.28 \%$ para as topologias Finlândia e NSFNet, respectivamente. Como sugestão para trabalhos futuros, pretende-se estudar os efeitos de uma otimização multiobjetivo para minimizar a probabilidade de bloqueio e o tempo de execução considerando o compromisso entre ambos.

\section{AGRADECIMENTOS}

Os autores agradecem à CNPQ por ter financiado este trabalho, a colaboração da UFPE e UFRPE.

\section{REFERÊNCIAS}

[1] Anja Feldmann, Oliver Gasser, Franziska Lichtblau, Eric Pujol, Igmar Poese, Christoph Dietzel, Daniel Wagner, et al. Implications of the covid-19 pandemic on the internet traffic. In Broadband Coverage in Germany; 15th ITG-Symposium, pages 1-5. VDE, 2021.

[2] Danilo Ricardo Barbosa de ARAÚJO. Planejamento de redes ópticas usando inteligência computacional e ciência das redes. 2015.

[3] Rajiv Ramaswami, Kumar Sivarajan, and Galen Sasaki. Optical networks: a practical perspective. Morgan Kaufmann, 2009.

[4] Masahiko Jinno, Hidehiko Takara, Bartlomiej Kozicki, Yukio Tsukishima, Yoshiaki Sone, and Shinji Matsuoka. Spectrum-efficient and scalable elastic optical path network: architecture, benefits, and enabling technologies. IEEE communications magazine, 47(11):66-73, 2009.

[5] AVS Xavier, RC Almeida, HA Pereira, AR Chaves, and CJA BastosFilho. Algoritmo de roteamento baseado na continuidade de formas de conexões em redes ópticas elásticas. Momag, 2014.

[6] José Cleyton da Silva, Carmelo JA Bastos-Filho, Isabella de S Couto, André VS Xavier, et al. Uma abordagem de otimização para o algoritmo de roteamento utilizando lógica nebulosa. 2018.

[7] Paulo Fernando de SOUZA FILHO. Proposta de heurística e metaheurística para alocação eficiente de recursos em redes com multiplexação por divisão espacial. Master's thesis, Universidade Federal de Pernambuco, 2018.

[8] RC Almeida Jr, Alex Ferreira dos Santos, KDR Assis, H Waldman, and JF Martins-Filho. Slot assignment strategy to reduce loss of capacity of contiguous-slot path requests in flexible grid optical networks. Electronics Letters, 49(5):359-361, 2013.

[9] Paulo F de Souza Filho and Raul C Almeida Jr. Redução da perda de capacidade em redes ópticas elásticas com multiplexação espacial. 2020.

[10] Clayton JN Lira, RC Almeida Jr, and Daniel AR Chaves. Estratégias de rsa utilizando split spectrum e o algoritmo mscl em eons. 2020.

[11] Clayton Natal Lira, Raul Almeida Júnior, Daniel Chaves, Helio Waldman, and Karcius Assis. Meta-heuristic procedure for enhanced spectrum fragmentation assessment in elastic optical networks. Journal of Communication and Information Systems, 35(1):333-337, 2020.

[12] Bijoy Chand Chatterjee, Nityananda Sarma, and Eiji Oki. Routing and spectrum allocation in elastic optical networks: A tutorial. IEEE Communications Surveys \& Tutorials, 17(3):1776-1800, 2015.

[13] Daniel AR Chaves, Helder A Pereira, Carmelo JA Bastos-Filho, and Joaquim F Martins-Filho. Simton: A simulator for transparent optical networks. Journal of Communication and Information Systems, 25(1), 2010.

[14] Jin Y Yen. Finding the $\mathrm{k}$ shortest loopless paths in a network. management Science, 17(11):712-716, 1971. 